"Supporting Information" for

\title{
Recyclable Thermosets Based on Dynamic Amidation and Aza-Michael Addition Chemistry
}

\author{
Ranjana Baruah, ${ }^{\dagger}$ Anuj Kumar, ${ }^{\dagger}$ Rewati Raman Ujjwal, ${ }^{\dagger}$ Soumya Kedia, ${ }^{\dagger}$ Amit Ranjan ${ }^{\ddagger} \&$ \\ Umaprasana Ojha*,†
}

†Department of Chemistry, Rajiv Gandhi Institute of Petroleum Technology Rae Bareli, UP229316, India

* Department of Chemical Engineering, Rajiv Gandhi Institute of Petroleum Technology Rae Bareli, UP-229316, India

Corresponding Author:

E mail: uojha@rgipt.ac.in,

Phone: 05352704221, Fax: 05352217456 


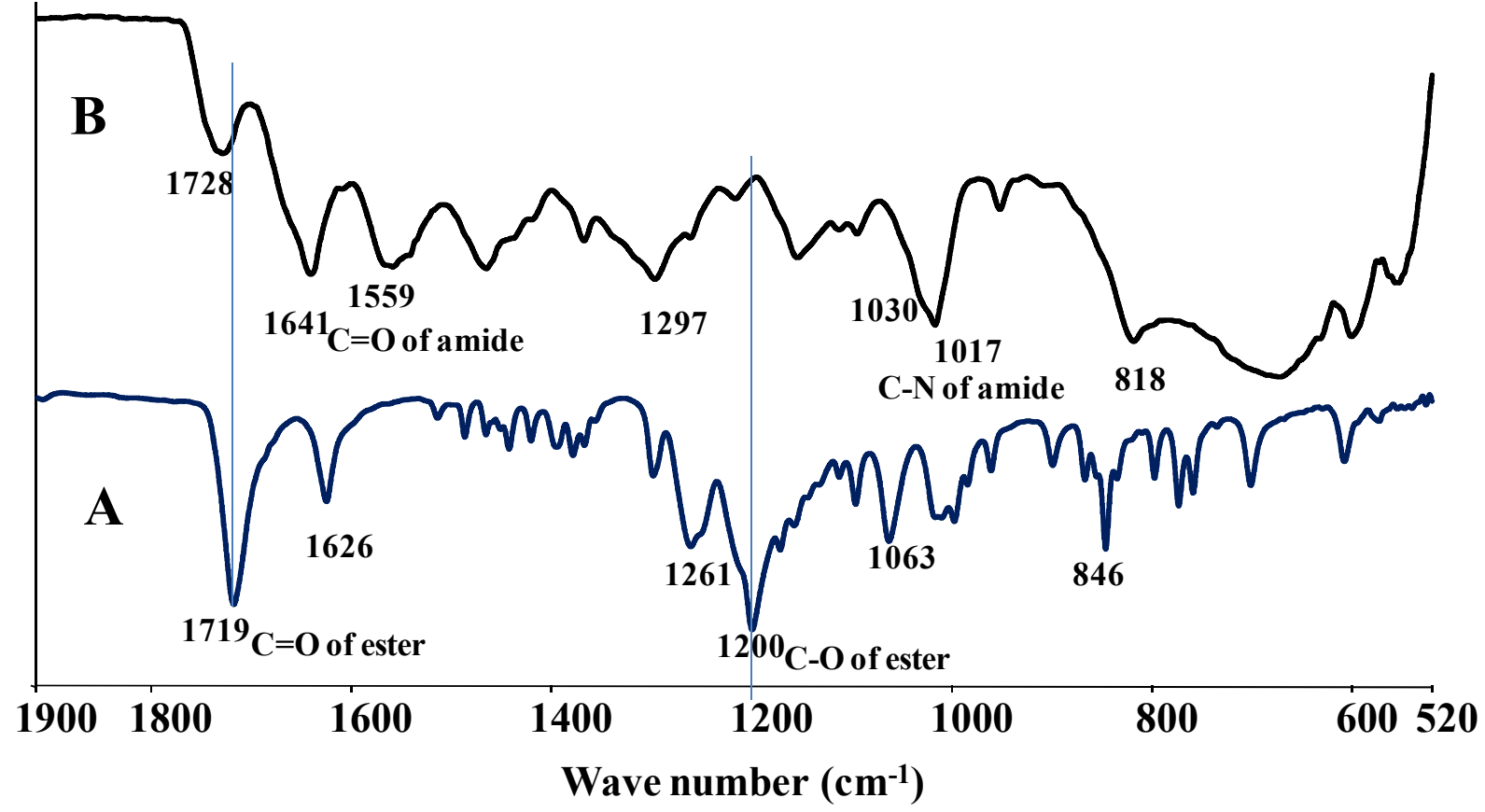

Figure S1: FTIR spectra of (A) TPMD and (B) TPMD-TETA-3 network 


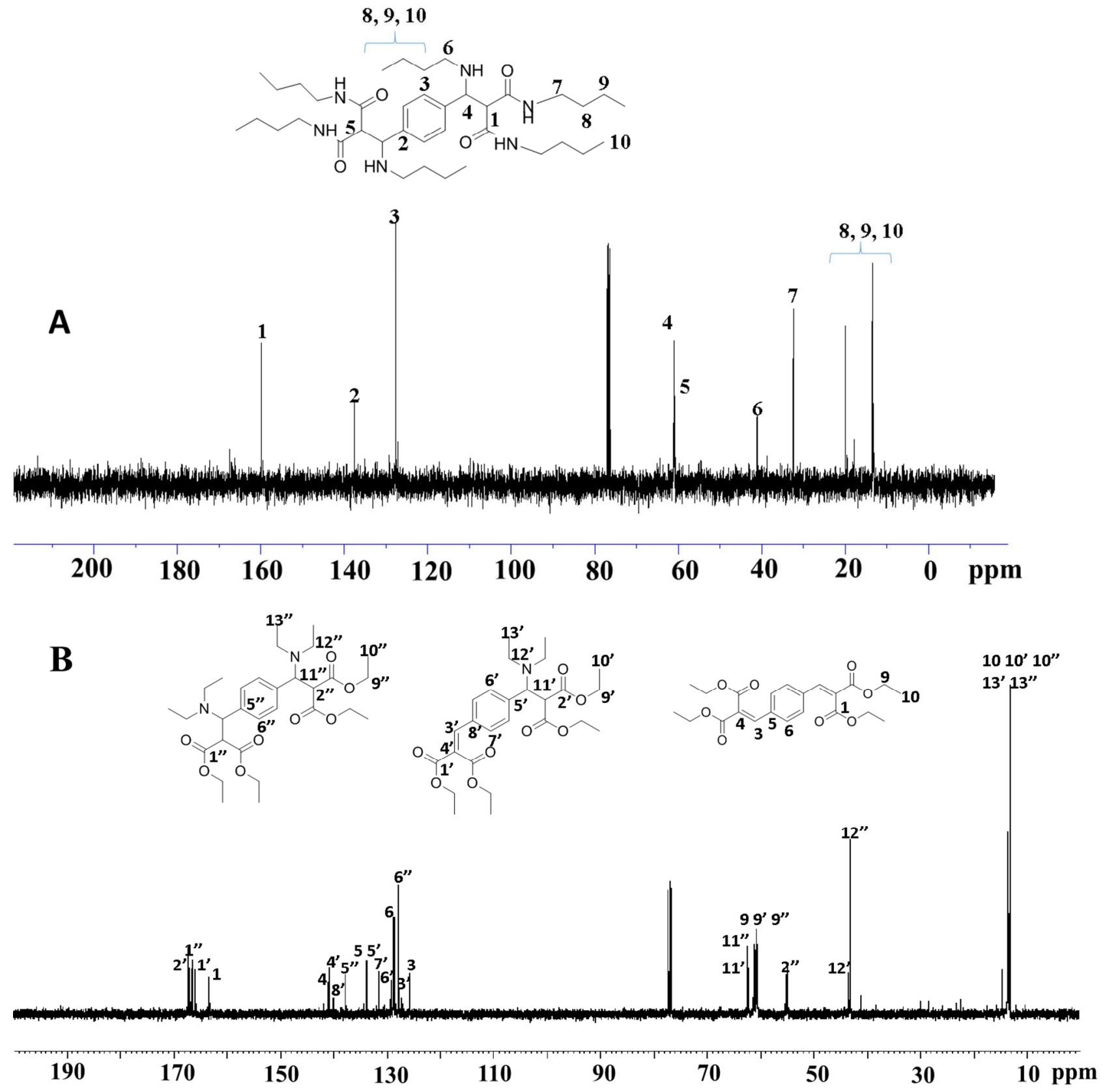

Figure S2: ${ }^{13} \mathrm{C}$ NMR spectra of (A) P-12 and (B) P-21 recorded in $\mathrm{CDCl}_{3}$ solvent 


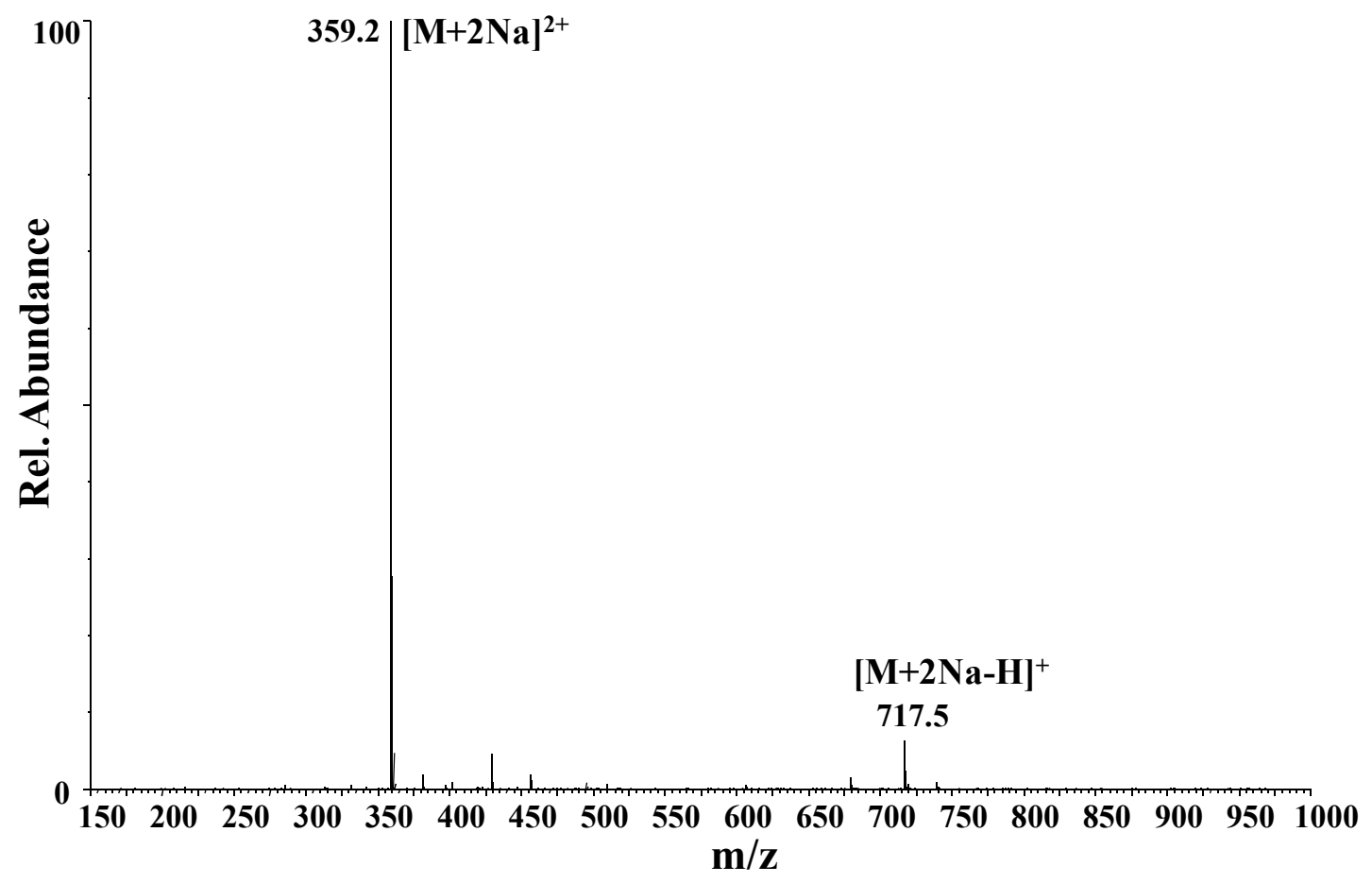

Figure S3: ESI-MS trace of P-12 (product of reaction between TPMD and n-butyl amine at 50 $\left.{ }^{\circ} \mathrm{C}\right)$ 


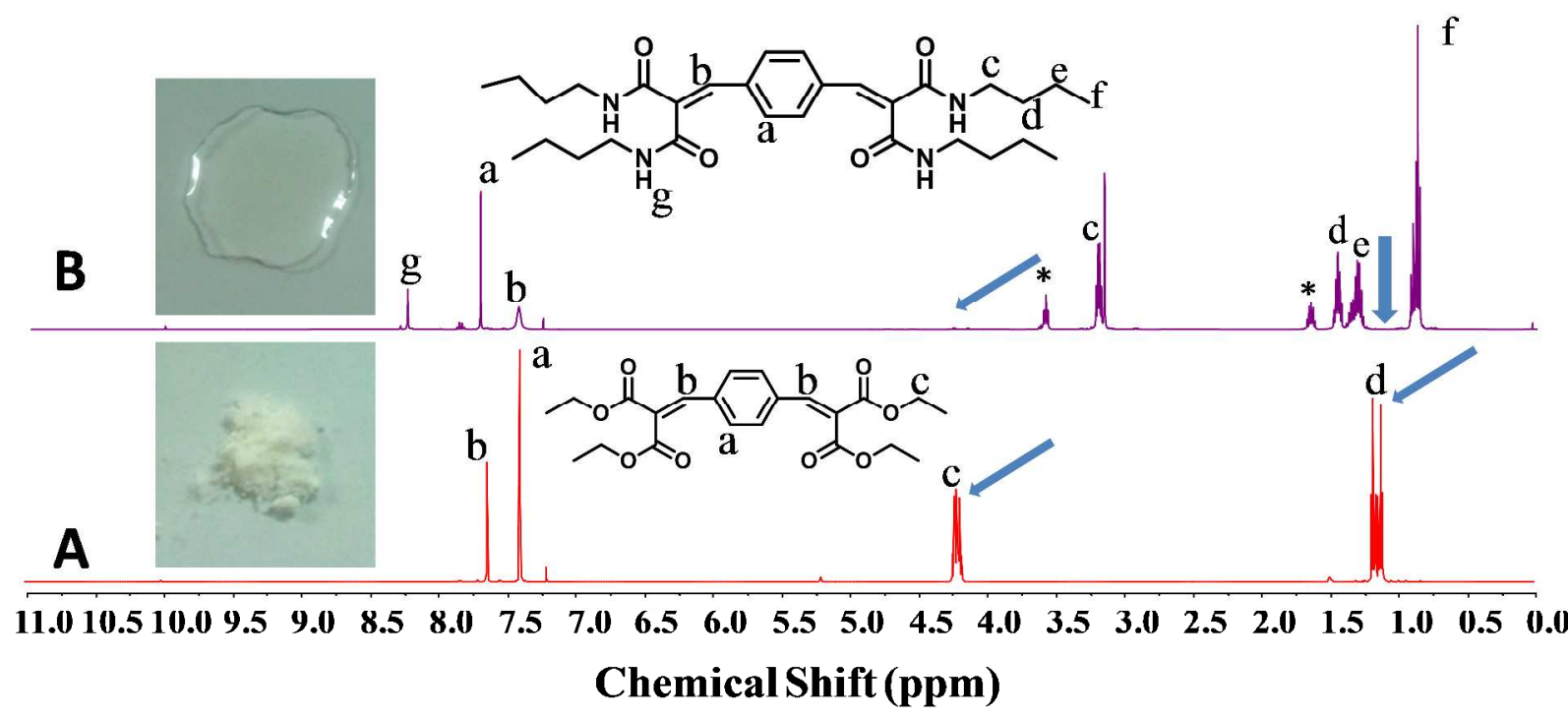

Figure S4: ${ }^{1} \mathrm{H}$ NMR spectra of (A) TPMD and (B) P-11 (product of reaction between TPMD and n-butyl amine at $10{ }^{\circ} \mathrm{C}$. The peaks marked with "*” is assigned to the small fraction of aza Michel adduct formed during the reaction

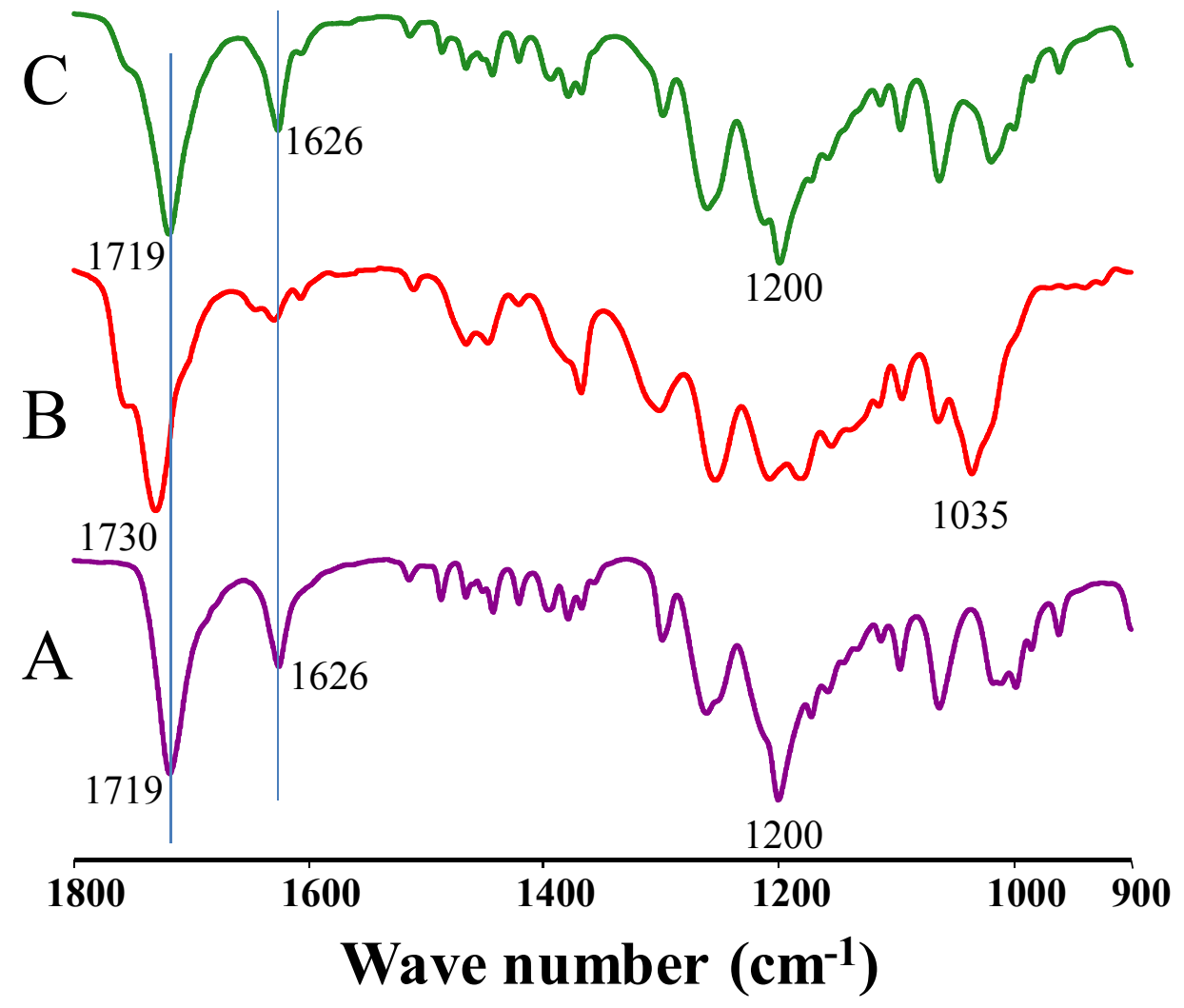

Figure S5: FTIR spectra of (A) TPMD, (B) product obtained after reacting TPMD with diethyl amine at $50{ }^{\circ} \mathrm{C}$ and $(\mathrm{C})$ product obtained after exposing product " $\mathrm{B}$ " to atmosphere for 48 $\mathrm{h}$ 


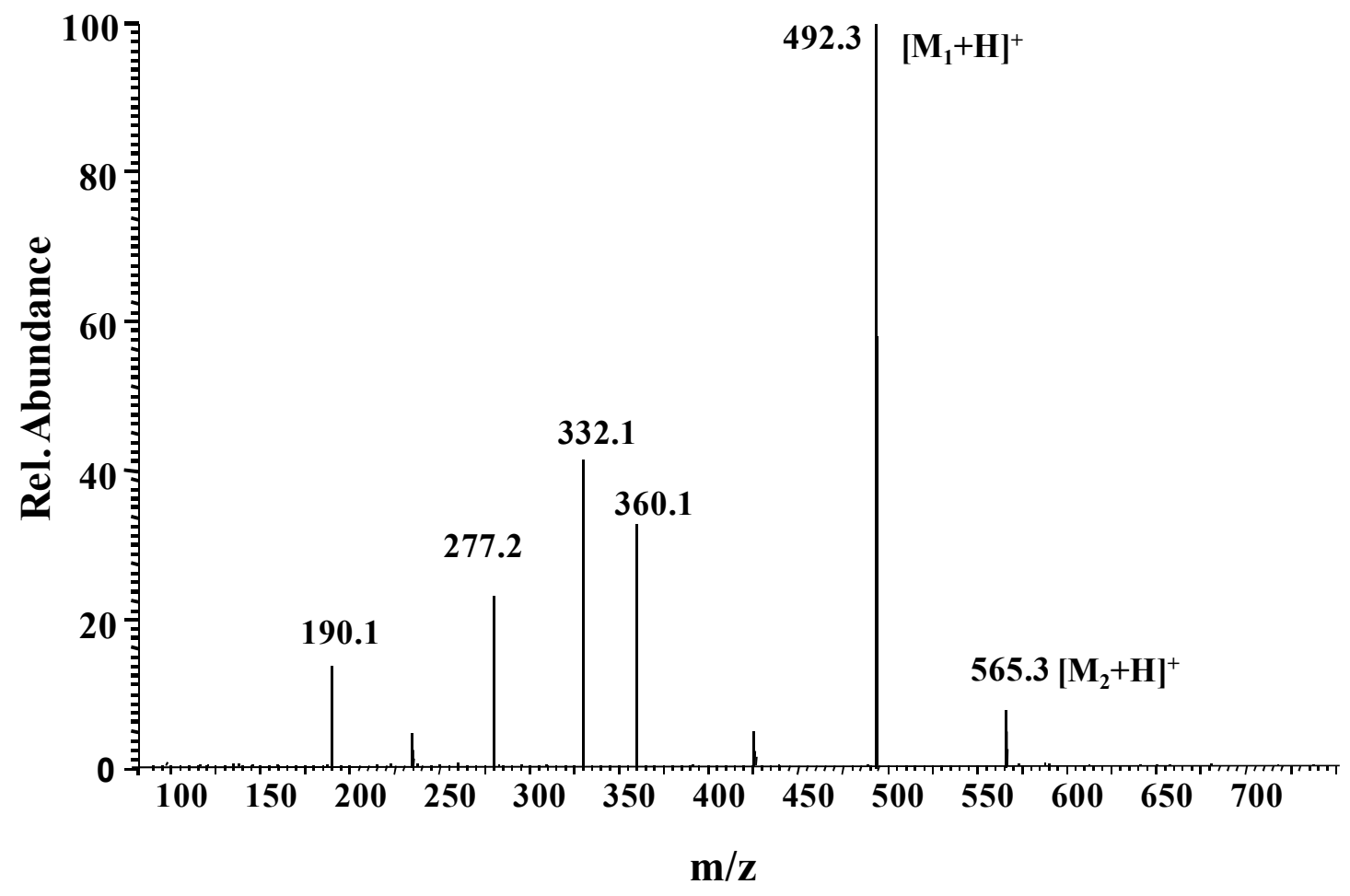

Figure S6: ESI-MS trace of P-21 (product of TPMD and diethyl amine at $50{ }^{\circ} \mathrm{C}$ )

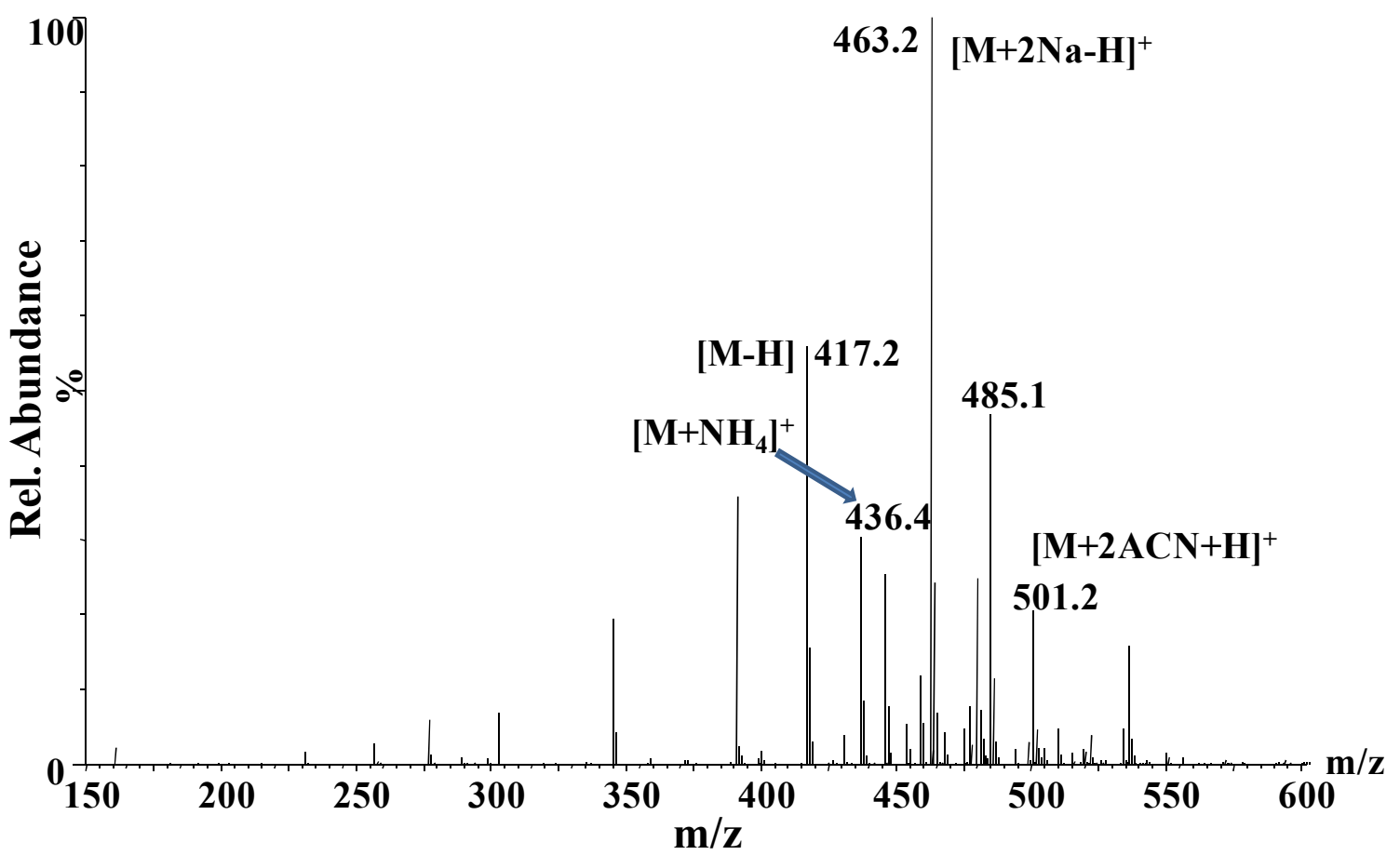

Figure S7: ESI-MS trace of the product obtained after exposing P-21 to atmosphere for $48 \mathrm{~h}$ 


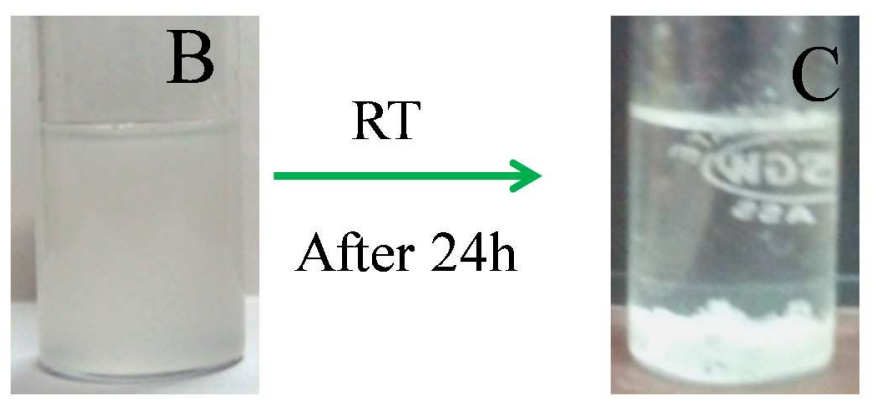

Figure S8: (B) Solution of P-12 in 0.1 molar acetic acid at $25^{\circ} \mathrm{C}$ after $1.5 \mathrm{~h},(\mathrm{C})$ the same solution after $24 \mathrm{~h}$

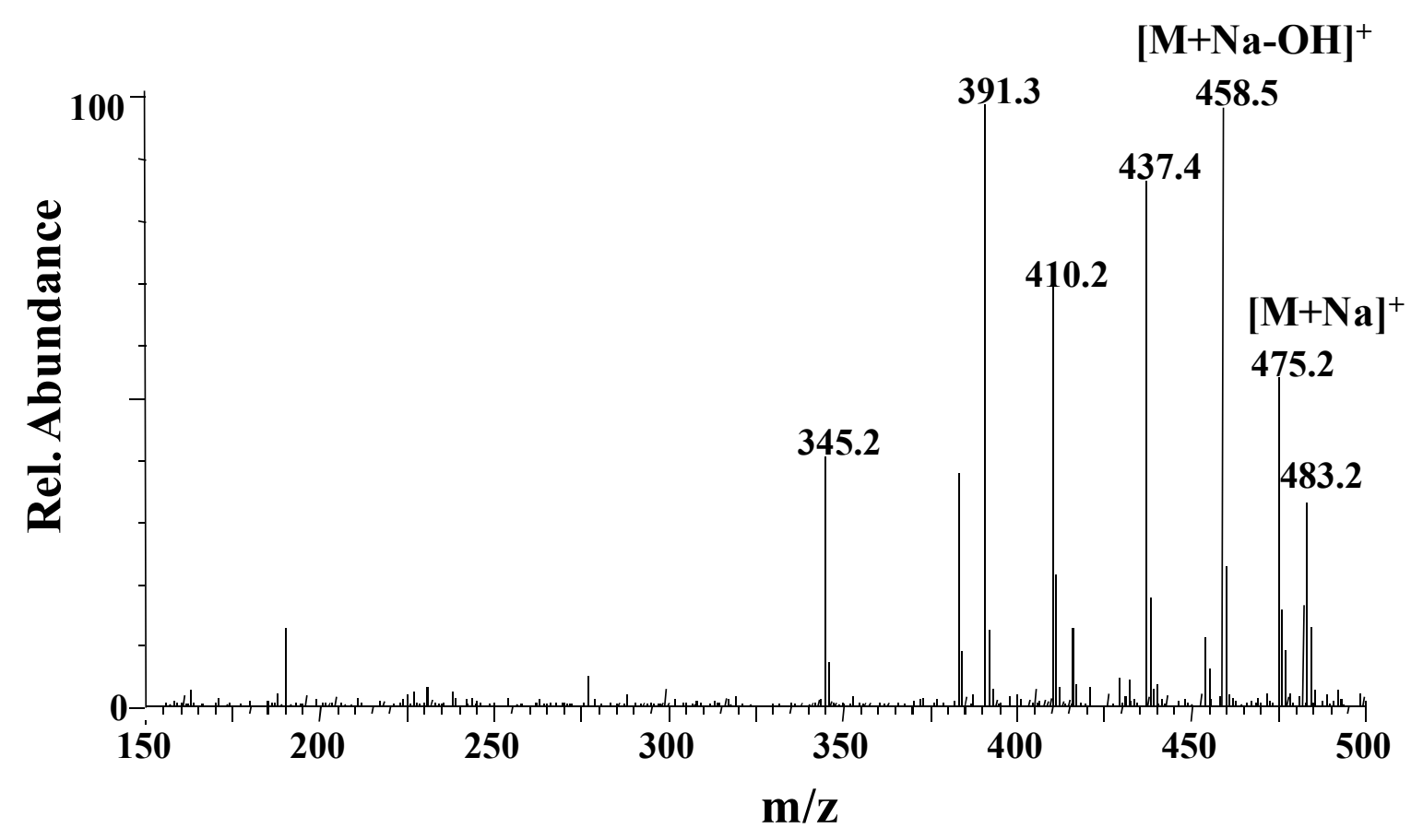

Figure S9: ESI-MS trace of P-13 (hydrolyzed product of P-12) 


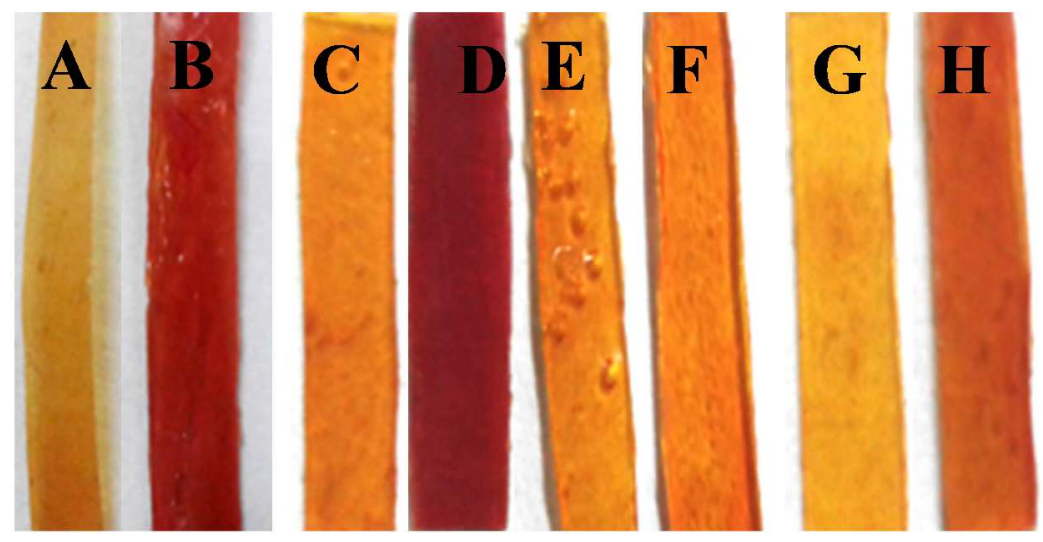

Figure S10: Photographs of thin strips of (A) TPMD-TETA-5, (B) TPMD-TETA-3, (C) TPMD-DETA-5, (D) TPMD-DETA-3, (E) TPMD-PEHA-5, (F) TPMD-PEHA-3, (G) TPMDTEPA-5 and (H) TPMD-TEPA-3.

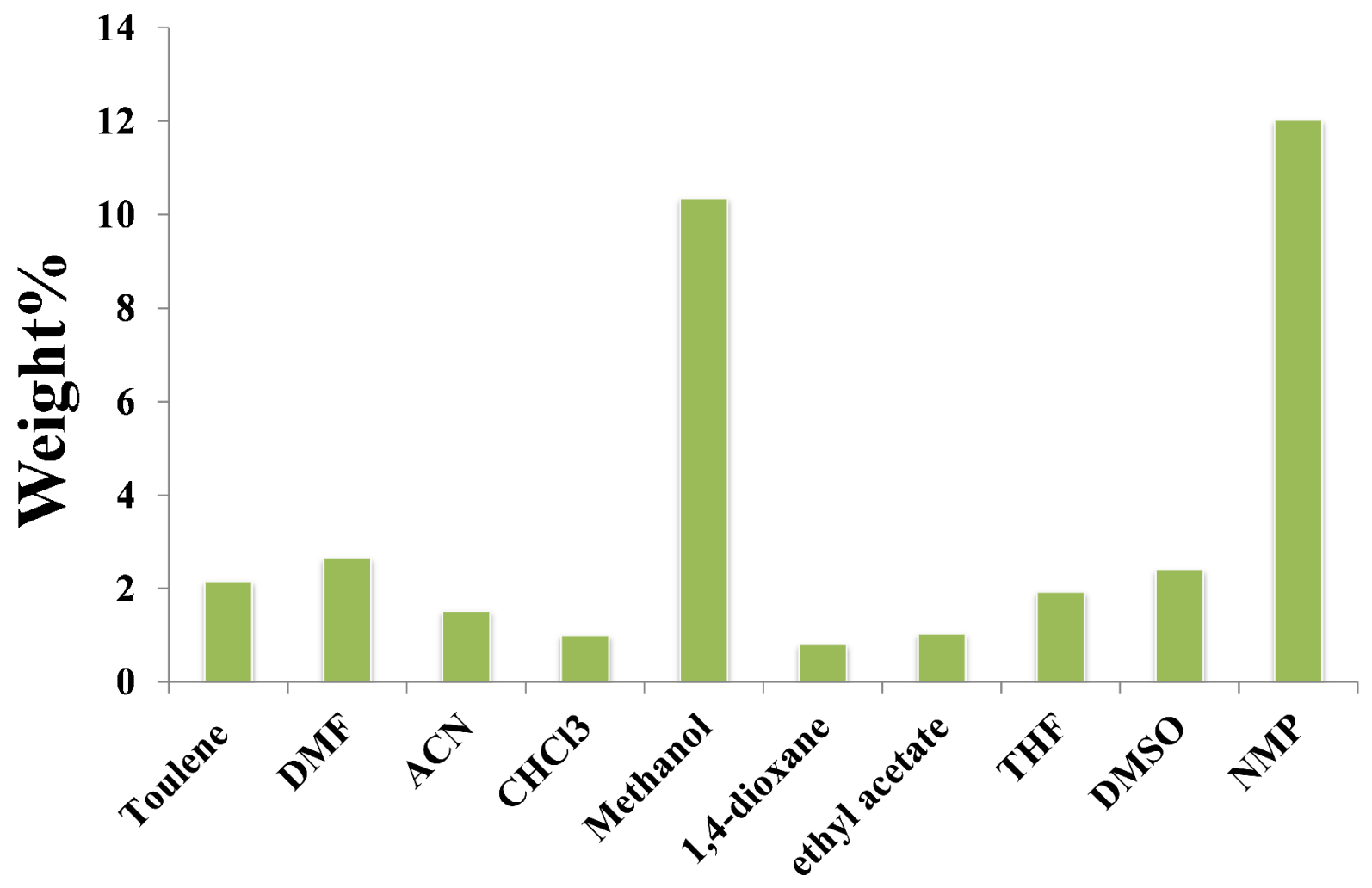

Figure S11: Swelling ratios (\% of the original weight) of TPMD-TETA-3 in different organic solvents 


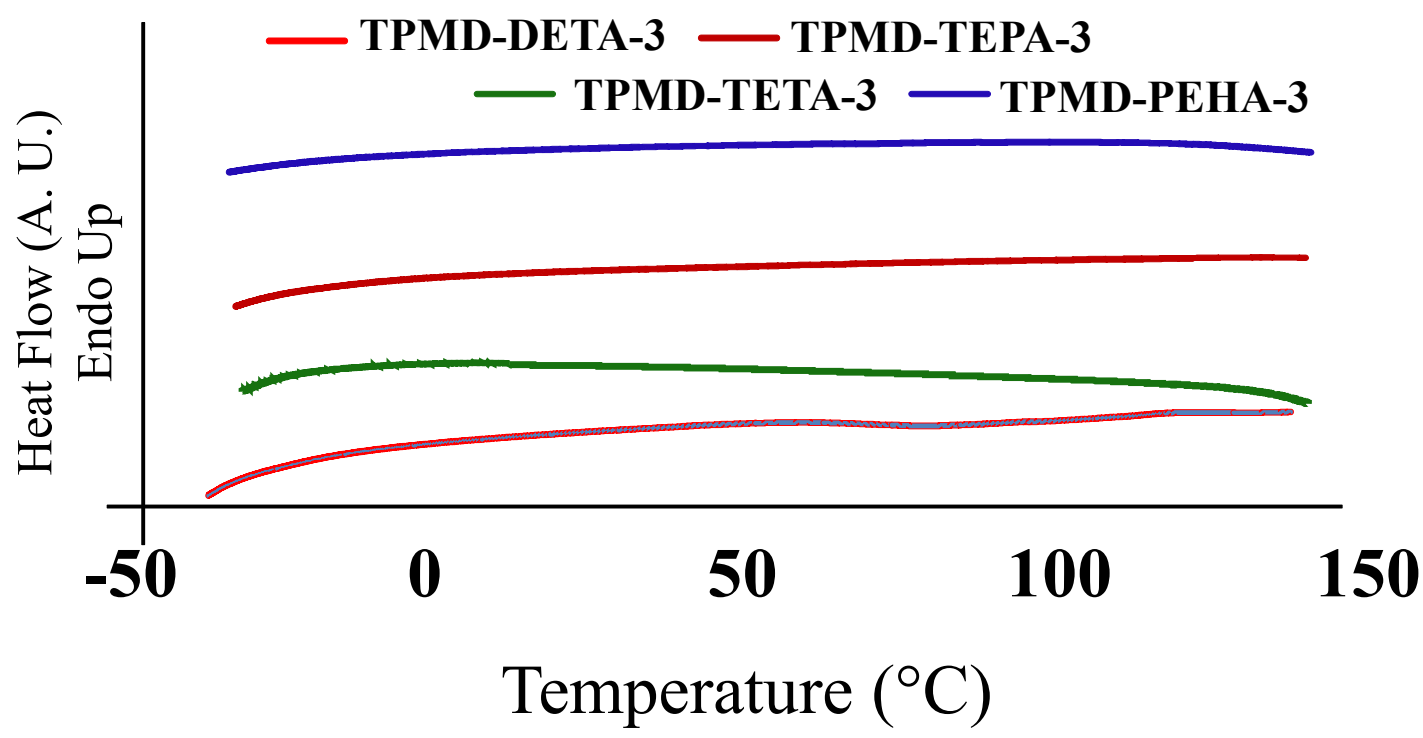

Figure S12: DSC second heating traces of the CANs possessing $30 \mathrm{~mol} \%$ of the TPMD recorded under $\mathrm{N}_{2}$ atmosphere

Amide I: $\mathbf{C}=\mathbf{O}$ stretch

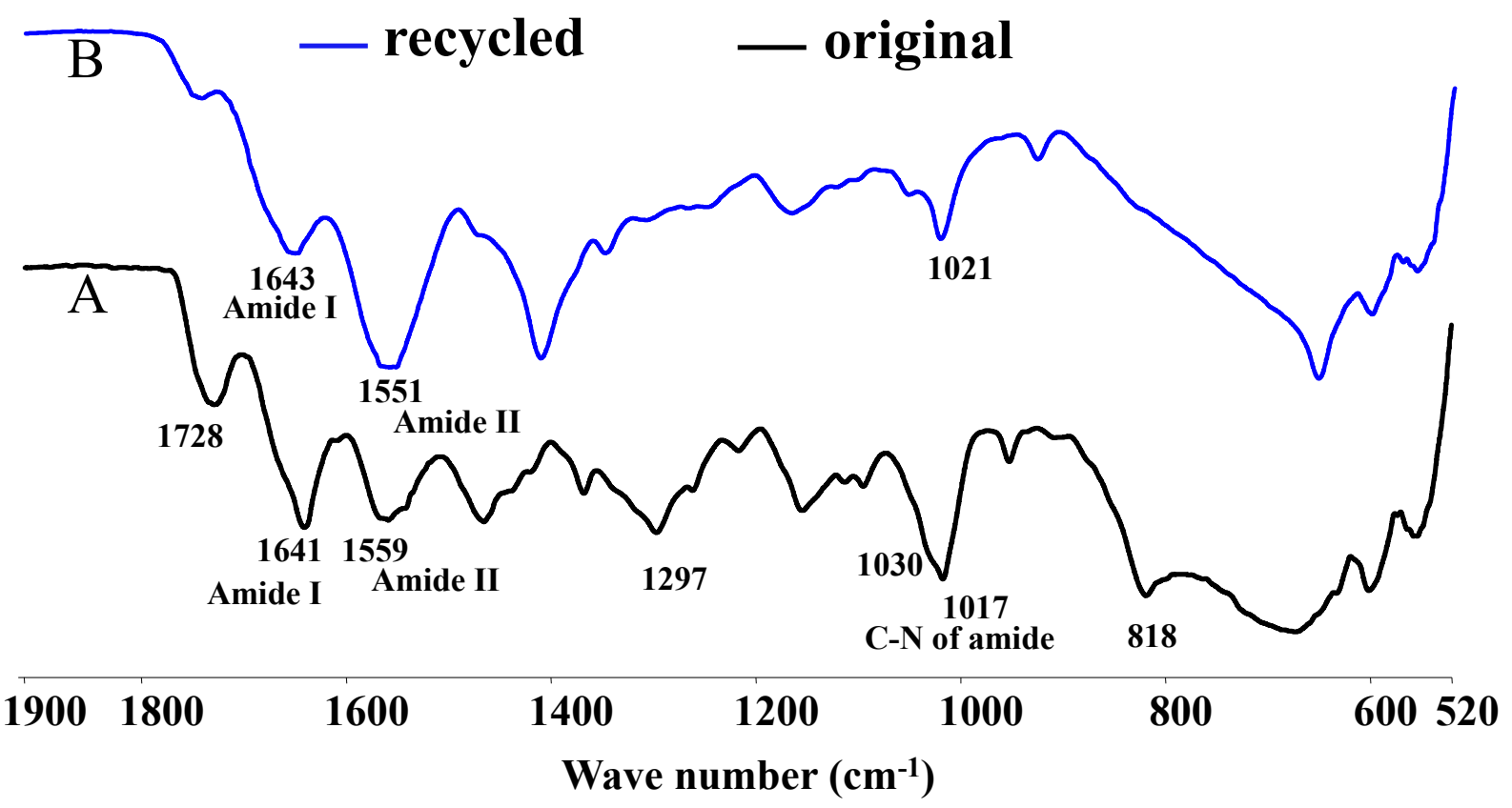

Figure S13: FTIR spectra of (A) original and (B) recycled TPMD-TETA-3 
Table S1: Tensile properties of the networks recorded as thin films under ambient conditions

\begin{tabular}{cccll}
\hline \multicolumn{1}{c}{ Code } & $\begin{array}{c}\text { [TPMD }] \\
(\mathrm{mol} \%)\end{array}$ & $\begin{array}{c}\text { UTS } \\
(\mathrm{MPa})^{*}\end{array}$ & $\begin{array}{c}\text { Elongation at } \\
\text { Break }(\%)\end{array}$ & $\begin{array}{c}\text { Young's } \\
\text { Modulus (MPa) }\end{array}$ \\
\hline TPMD-TETA-5 & 50 & $21.9 \pm 3.8$ & $3.7 \pm 0.5$ & $1680 \pm 50$ \\
TPMD-TETA-3 & 30 & $27.3 \pm 2.0$ & $4.4 \pm 0.5$ & $2080 \pm 200$ \\
TPMD-DETA-5 & 50 & $3.6 \pm 0.5$ & $2.5 \pm 0.4$ & $507 \pm 100$ \\
TPMD-DETA-3 & 30 & $22.8 \pm 2.0$ & $2.6 \pm 0.8$ & $1980 \pm 100$ \\
TPMD-PEHA-5 & 50 & $18.1 \pm 2.4$ & $2.7 \pm 0.5$ & $1225 \pm 130$ \\
TPMD-PEHA-3 & 30 & $30.9 \pm 2.0$ & $2.5 \pm 1.8$ & $2990 \pm 220$ \\
TPMD-TEPA-3 & 30 & $35.5 \pm 3.0$ & $1.7 \pm 1.0$ & $3035 \pm 250$ \\
TPMD-TEPA-2 & 50 & $5.8 \pm 0.6$ & $15.0 \pm 1.7$ & $164 \pm 50$ \\
\hline
\end{tabular}

*The data is presented with standard deviation values.

Table S2: TGA data of the CANs possessing $30 \mathrm{~mol} \%$ of TPMD

\begin{tabular}{ccc}
\hline Code & $\begin{array}{c}\mathrm{T}_{10} \text { (Temperature at } \\
10 \% \text { weight loss) }\end{array}$ & $\begin{array}{c}\text { Char yield (remaining weight } \\
\left.\text { after } 700^{\circ} \mathrm{C}\right)(\%)\end{array}$ \\
\hline TPMD-DETA-3 & 215 & 18 \\
TPMD-TETA-3 & 212 & 36 \\
TPMD-TEPA-3 & 220 & 23 \\
TPMD-PEHA-3 & 200 & 38 \\
\hline
\end{tabular}


Table S3: Tensile properties of the as synthesized and recycled networks. All the networks have $30 \mathrm{~mol} \%$ of TPMD

\begin{tabular}{ccccccc}
\hline \multirow{2}{*}{ Code } & \multicolumn{5}{c}{$\begin{array}{c}\text { As prepared } \\
\text { (control) }\end{array}$} \\
\cline { 2 - 7 } & UTS & $\begin{array}{c}\text { Elongation } \\
\text { at break } \% *\end{array}$ & $\begin{array}{c}\text { Modulus } \\
(\mathrm{MPa})^{*}\end{array}$ & $\begin{array}{c}\text { UTS } \\
(\mathrm{MPa})^{*}\end{array}$ & $\begin{array}{c}\text { Elongation at } \\
\text { break } \% *\end{array}$ & $\begin{array}{c}\text { Modulus } \\
(\mathrm{MPa})^{*}\end{array}$ \\
\hline TPMD-TETA-3 & $27.3 \pm 2.0$ & $4.45 \pm 0.5$ & $2080 \pm 200$ & $26.6 \pm 1.5$ & $3.85 \pm 0.3$ & $1975 \pm 65$ \\
& & & & & & \\
TPMD-DETA-3 & $22.8 \pm 2.0$ & $2.57 \pm 0.8$ & $1980 \pm 100$ & $21.5 \pm 1.0$ & $2.68 \pm 0.5$ & $1950 \pm 50$ \\
& & & & & & \\
TPMD-PEHA-3 & $30.9 \pm 2.0$ & $2.56 \pm 1.8$ & $2990 \pm 220$ & $30.8 \pm 1.5$ & $1.28 \pm 0.3$ & $3080 \pm 100$ \\
& & & & & & \\
TPMD-TEPA-3 & $35.5 \pm 3.0$ & $1.72 \pm 1.0$ & $3035 \pm 250$ & $34.3 \pm 1.0$ & $1.45 \pm 0.5$ & $3059 \pm 200$ \\
& & & & & & \\
\hline
\end{tabular}

\footnotetext{
*the data is presented with the standard deviation values
} 